Ann. Biol. anim. Bioch. Biophys., I976, 16 (6), 879-89o.

\title{
LES TANINS DES GRAINES DE SORGHO. IMPORTANCE DANS L'UTILISATION DIGESTIVE DE L'AZOTE CHEZ LE RAT EN CROISSANCE
}

\author{
Josette MARTIN-TANGUY, M. VERMOREL, M. LENOBLE et C. MARTIN \\ avec la collaboration technique de M. GalleT \\ Station de Physiopathologie végétale, \\ Centre de Recherches de Dijon, I. N. R. A., \\ B.V. 1540, 21034 Dijon \\ Laboratoire d'Étude du Métabolisme énergétique, \\ Centre de Recherches de Clermont-Ferrand, I. N.R. A,, \\ Theix, Saint Genès Champanelle, 63110 Beaumont \\ Station d'Amélioration des Plantes fourragères, I. N. R. A., \\ 86600 Lusignan

\section{RÉSUMÉ,}

Les téguments de nombreuses variétés de sorgho renferment des tanins condensés formés de leucocyanidine. Ces procyanidines dépriment l'utilisation digestive de l'azote chez le Rat en croissance ; in vitro, la présence de ces substances est vraisemblablement responsable d'un effet dépressif sur la diminution de la matière sèche après " digestion enzymatique ».

\section{INTRODUCTION}

Les tanins contenus dans les graines de certaines variétés de sorgho confèrent à ces plantes quelques avantages agronomiques telles la résistance à l'attaque de prédateurs comme les oiseaux, 1'inhibition de la germination des graines avant récolte, et une résistance importante aux intempéries (HARRIs, I969). Cependant, les tanins apparaissent responsables chez les animaux monogastriques et les ruminants d'une mauvaise utilisation digestive des graines de sorgho.

Chang et Fuller (I964) observent chez des poulets un retard de croissance lors de l'introduction dans les régimes alimentaires de graines de variétés de sorgho riches en tanins. McGinry (Ig68) note chez les animaux ruminants que les variétés 
de sorgho à hautes teneurs en tanins ont une digestibilité des matières sèches inférieure à celle présentée par des graines de variétés de sorgho pauvres en tanins.

Les résultats présentés concernent la structure chimique des tanins présents dans les graines de sorgho et l'effet dépressif de certaines de ces substances sur l'utilisation digestive des protéines chez le Rat en croissance et sur la disparition in vitro de la matière sèche.

\section{MATÉRIEL ET MÉTHODES}

\section{I. - Matériel. Préparation des extraits}

\section{I) Matériel.}

Les échantillons de sorgho utilisés dans les études sur Rats correspondent à des variétés récoltées en 1967, I970 et 1971 dans les départements de l'Hérault et de l'Aude. Ils ont été fournis par la Station d'Amélioration des Plantes du Centre de Recherches de Montpellier ou par la Coopérative Agricole Lauragaise de Castelnaudary dans 1'Aude. Les caractéristiques de ces sorghos sont indiquées dans les tableaux 2 et 3 .

L'hybride "INRA 450 » a été obtenu à partir de 2 lignées américaines (A 3052 et SD I02) ; il est inscrit au Catalogue Officiel des Espèces et Variétés depuis janvier 1969. Les variétés NK 120, NK 222 et AKS 614 sont d'origine américaine. Les sorghos utilisés dans l'étude de la diminution de la matière sèche in vitro après " digestion enzymatique " sont des hybrides (lignées $\mathrm{F}_{3}$ ) sélectionnés à la Station d'Amélioration des Plantes fourragères de Lusignan. Ils proviennent de croisements entre des " sorghos sauvages " précoces et la lignée américaine B. Martin.

2) Préparation des extraits.

Les graines sont broyées au mixer (Sorvall) dans du méthanol à 50 p. roo. Les extraits sont mis sur agitateur magnétique puis centrifugés. L'opération est renouvelée trois fois. Les surnageants sont réunis. L'alcool est éliminé sous vide dans un évaporateur rotatif. La solution résiduelle est débarrassée des chlorophylles et caroténoïdes par l'éther de pétrole. L'extrait aqueux est ensuite mis à sec sous vide et amené à la concentration désirée par du méthanol à 50 p. Ioo.

\section{II. - Dosage, fractionnement et identification des tanins}

\section{I) Dosage.}

Le dosage des tanins condensés du sorgho (ou proanthocyanidines) repose sur la transformation de ces substances en anthocyanidines colorées par un chauffage en milieu acide (SwalN et Hillis, I959). Les déterminations colorimétriques sont faites à $550 \mathrm{~nm}$ pour la cyanidine selon les techniques décrites par Swain et Hillis (1959). Étant donné les difficultés de rapporter les résultats à ceux obtenus à partir d'un produit de référence, dont on n'est jamais certain que la constitution soit identique à celle du tanin étudié, de nombreux auteurs préfèrent exprimer en densités optiques les quantités de tanins et définissent ainsi une grandeur (LA) (TroYer, I964; Quesnex, I968, Bate Smith, 1973). Dans ce travail les concentrations en proanthocyanidines seront exprimées sous cette forme.

La détermination d'un indice lié à la polymérisation moyenne des proanthocyanidines est réalisée selon la méthode de GoldSTEIN et SwaIN (I963). Cet indice est obtenu en soumettant l'échantillon à deux réactions chimiques. Ces réactions sont la transformation des leucoanthocyanidines en anthocyanidines (LA), et la formation d'une combinaison colorée avec la vanilline en milieu acide (grandeur $\mathrm{V}$ ). La vanilline réagit avec les carbones 6 ou 8 des molécules de flavanes intervenant dans la structure des tanins condensés. Ainsi, chaque fois qu'une molécule de flavane est engagée par ses carbones 6 ou 8 dans un polymère, elle ne réagit pas avec l'aldéhyde. La grandeur V est mesurée à $500 \mathrm{~nm}$. L'indice est exprimé par le rapport V/LA.

\section{2) Fractionnement.}

Le fractionnement des proanthocyanidines est réalisé selon les techniques préconisées par QuesNel (1968). Les tanins condensés extraits au méthanol absolu et précipités par des volumes croissants d'éther éthylique sont séparés en trois fractions ( $A, B$ et $C$ ). Le procédé de fractionnement est donné par le tableau I. 


\section{TABLFAU I}

Fractionnement des proanthocyanidines (selon QUESNEL 1968)

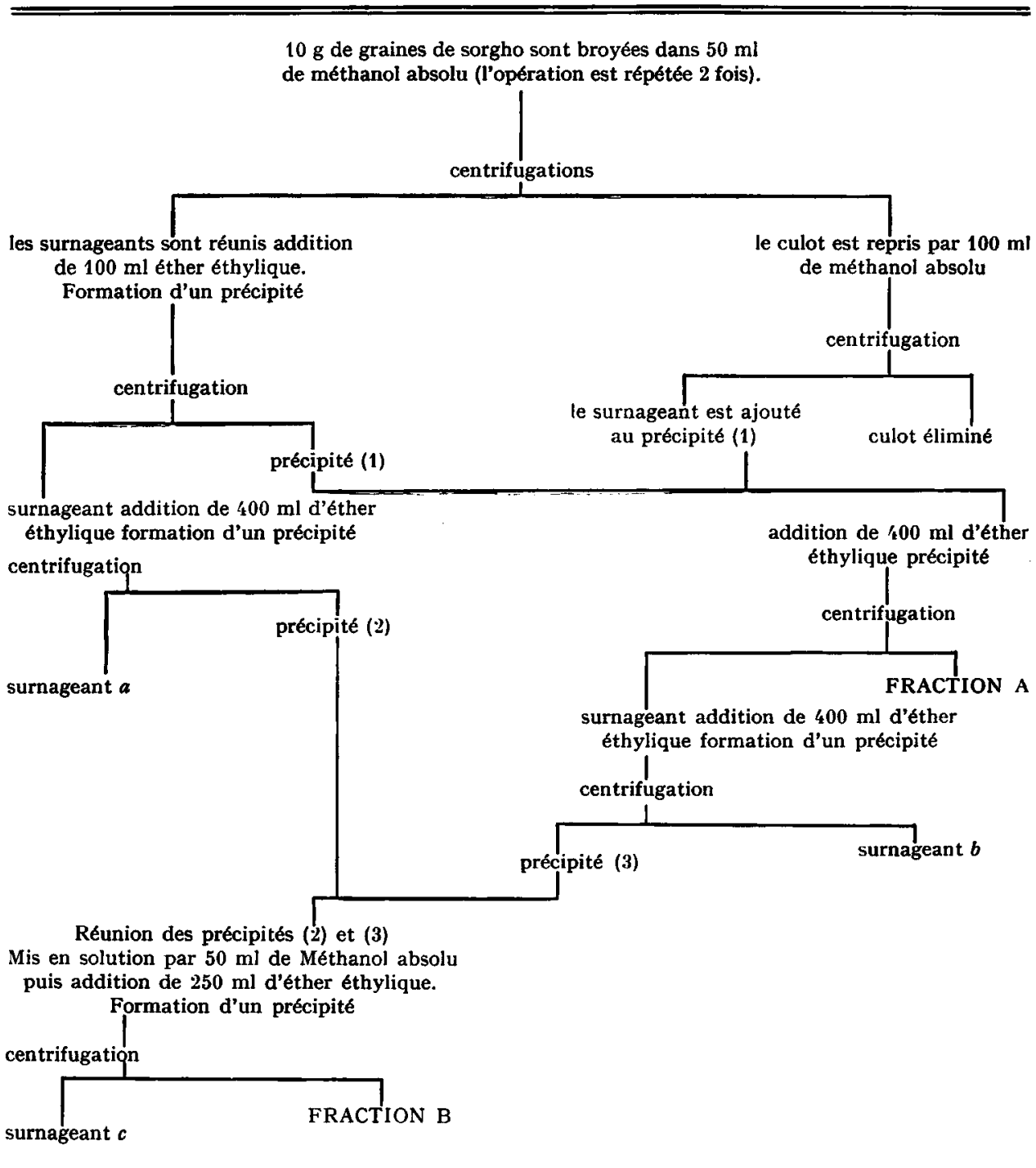

Les surnageants $(a b c)$ sont réunis mis à sec; on reprend par $20 \mathrm{ml}$ de méthanol ; addition de $200 \mathrm{ml}$ d'éther éthylique. Le précipité est centrifugé $: \longrightarrow$ FRACTION $\mathrm{C}$

\section{3) Chromatographie.}

Les proanthocyanidines et leurs produits de transformation (flavanol-3, anthocyanidines) sont séparés et identifiés par chromatographie sur papier ou sur plaques de cellulose. La séparation des flavanol-3 et flavanediols-3, 4 est effectuéc par chromatographie à deux dimensions sur 
papier en utilisant le mélange butanol-acide acétique-eau (4-I-5 V/V) en première dimension et de 1'acide acétique à 2 p. Ioo en deuxième dimension (QuESNEL, I968). La révélation de ces composés est réalisée par le réactif à la vanilline chlorhydrique (GoldsTein et SwaIn, I963).

La séparation et l'identification des anthocyanidines sont faites soit par chromatographie sur papier en utilisant le butanol acétique ou le Forestal (acide acétique, $\mathrm{HCl}$ concentré, eau ; 30, 3, io V/V (Bate-Smith et Swain, I967; Bate-Smith, r969), soit par chromatographie sur plaques de cellulose en utilisant un solvant très acide (acide formique, $\mathrm{HCl} 25 \mathrm{p}$. Ioo, eau ; ro, I, $3 \mathrm{~V} / \mathrm{V}$ (VAN BRAGT, 1966). Les mesures spectrales sont réalisées après élution en milieu méthanol chlorhydrique.

\section{4) Hydrolyse ménagée.}

Quinze ml d'une solution o,2 $\mathrm{N}$ HCl sont chauffés sous reflux; au temps zéro, il est ajouté I $5 \mathrm{ml}$ d'une solution aqueuse contenant la fraction des tanins à étudier (Ioo $\mathrm{mg}$ ). Des prises d'essais sont effectuées après ro secondes, $3,6,9,12,30$ et 60 minutes. Les extraits sont refroidis et chromatographiés.

5) Hydrolyse forte.

A $5 \mathrm{ml}$ d'une solution aqueuse des tanins ( $15 \mathrm{mg}$ ) on ajoute $5 \mathrm{ml}$ d'une solution d'acide chlorhydrique $2 \mathrm{~N}$. Après chauffage à I $^{\circ} \mathrm{C}$ pendant $3^{\circ}$ minutes, les anthocyanidines sont extraites par l'alcool isoamylique et chromatographiées.

6) Réaction avec la gélatine (QUESNEL, 1968).

Cinquante $\mathrm{mg}$ de chaque fraction sont dissous dans $50 \mathrm{ml}$ d'eau. $\mathrm{r} \mathrm{ml}$ d'une solution de gélatine à I p. Ioo est ajouté goutte à goutte aux extraits contenant les tanins. La présence de substances présentant des propriétés de tanin se traduit par la formation d'un précipité avec la gélatine.

\section{III. - Essais sur l'utilisation digestive du sorgho chez le Rat en croissance}

Les études ont été réalisées en I968, I970 et 1972 (VERMOREL, I970).

\section{I) Animaux.}

Des rats mâles de race Sprague Dawley pesant $120 \mathrm{~g}$ environ sont répartis en lots comparables de 6 à 7 animaux et placés individuellement dans des cages à digestibilité.

\section{2) Régimes.}

Le sorgho constitue la principale source d'énergie ( 84 à 88 p. 10o) et d'azote (6o à 74 p. Ioo) des régimes expérimentaux. De la farine de harengs de Norvège et des acides aminés industriels sont ajoutés aux régimes en quantités variables, selon la teneur en tanins des sorghos et la digestibilité présumée de l'azote. Cela en vue d'assurer des apports voisins de matières azotées digestibles et de satisfaire les besoins en acides aminés indispensables du Rat en croissance (tabl. 2). Les régimes sont également supplémentés en minéraux, vitamines et acides gras essentiels. Ils sont distribués aux Rats sous forme humide dans des mangeoires spéciales permettant d'éviter le gaspillage.

\section{3) Mesures.}

Après séchage de l'aliment en étuve ventilée, les quantités d'aliment distribuées, refusées et consommées par chaque rat sont déterminées chaque jour.

Après une période d'adaptation de 4 jours aux régimes expérimentaux et aux cages à digestibilité, les fèces et l'urine sont collectées séparément pendant une période de ro jours. L'urine acidifiée et les fèces sont conservées à une température de - I $5^{\circ} \mathrm{C}$. Pour le premier lot, les échantillons de fèces et d'urine ont été analysés individuellement (VERMorel 197o). Dans les autres lots, les fèces des rats qui ont présenté des consommations voisines sont regroupées, lyophilisées puis mélangées en vue du dosage en triple de l'azote et de l'énergie brute sur l'échantillon moyen ainsi constitué. On procède de même dans le cas de l'urine (VERMOREL, I973).

Les mesures d'énergie brute ingérée, d'énergie métabolisée, d'azote ingéré, d'azote absorbé permettent de calculer pour le régime les coefficients d'utilisation digestive apparente de l'énergie 
(CUDaE) et de l'azote (CUDaN). A partir des coefficients d'utilisation digestive apparente de l'énergie et de l'azote des régimes, nous estimons les coefficients d'utilisation digestive du sorgho en supposant que la digestibilité des autres constituants du régime n'a pas été modifiée par la présence des tanins. Le CUDaE de la farine de poisson est pris égal à 88 et le CUDaE de l'huile de maïs et du mélange vitaminique égal à 98 , d'après les nombreux résultats antérieurs obtenus au laboratoire.

Deux autres coefficients sont calculés :

- le CUP $=$ coefficient d'utilisation pratique de l'azote.

- le $\mathrm{CR}=$ coefficient de rétention azotée.

TABLEAU 2

Composition des sorghos et des régimes utilisés

\begin{tabular}{|c|c|c|c|c|c|c|c|}
\hline Echantillon de Sorgho & INRA 450 & NK 222 & INRA 450 & INRA 450 & NK 120 & INRA 450 & AKS 614 \\
\hline $\begin{array}{c}\text { Année } \\
\text { Origine (référence) } \\
(\mathrm{N} \times 6,25) \text { p. } 100 \mathrm{MS}\end{array}$ & $\begin{array}{l}1967 \\
\text { C.A.L.* } \\
14,1\end{array}$ & $\begin{array}{c}1970 \\
\text { E.N.S.A.** } \\
8,9\end{array}$ & $\begin{array}{l}1971 \\
\text { E.N.S.A. } \\
13,2\end{array}$ & $\begin{array}{l}1970 \\
\text { E.N.S.A. } \\
11,4\end{array}$ & $\begin{array}{c}1970 \\
\text { C.A.L. } \\
11,1\end{array}$ & $\begin{array}{c}1971 \\
\text { C.A.L. } \\
10,2\end{array}$ & $\begin{array}{l}1970 \\
\text { E.N.S.A. } \\
11,3\end{array}$ \\
\hline \multicolumn{8}{|l|}{$\begin{array}{l}\text { Composition du régime } \\
\text { (g MS) }\end{array}$} \\
\hline Sorgho $\ldots \ldots \ldots \ldots \ldots$ & 880 & 940 & 957 & 950 & 950 & 910 & 900 \\
\hline Farine de poisson $\ldots \ldots$. & 40 & 46 & 19 & 36 & 36 & 75 & 80 \\
\hline Méthionine $\ldots \ldots \ldots \ldots$. & 0,8 & 2,0 & 2,0 & 2,0 & 2,0 & 2,0 & $\underline{2,0}$ \\
\hline L. Lysine $\ldots \ldots \ldots \ldots$ & 6,6 & 6,0 & 6,0 & 6,0 & 6,0 & 6,0 & 6,0 \\
\hline L. Thréonine $\ldots \ldots \ldots \ldots$ & 2,0 & 1,0 & 1,0 & 1,0 & 1,0 & 1,0 & 1,0 \\
\hline L. Tryptophane ........ & 0 & 0,5 & 0,5 & 0,5 & 0,5 & 0,5 & 0,5 \\
\hline L. Isoleucine $\ldots \ldots \ldots \ldots$ & 3,0 & 0 & 0 & 2,5 & 5,0 & 0 & 0 \\
\hline Mélange minéral $\ldots \ldots \ldots$ & 30 & 35 & 40 & 45 & 45 & 35 & 35 \\
\hline Mélange vitaminique $\ldots$. & 20 & 20 & 20 & 20 & 20 & 20 & 20 \\
\hline Huile de maïs vitaminisée. & 20 & 20 & 20 & 20 & 20 & 20 & 20 \\
\hline$(\mathrm{N} \times 6,25)$ p. 100 MS $\ldots$ & 15,4 & 12,0 & 14,1 & 13,7 & 13,6 & 15,0 & 16,5 \\
\hline Matières azotées digestibles & & & & & & & \\
\hline (p. 100 MS) $\ldots \ldots \ldots$ & 13,5 & 9,7 & 8,7 & 8,8 & 7,6 & 8,7 & 8,2 \\
\hline
\end{tabular}

* C.A.L. : Cooperative Agricole Lauragaise, Castelnaudary (Aude).

** E.N.S.A. : Station d'Amélioration des Plantes, École nationale supérieure agronomique de Montpellier (Hérault).

\section{IV. - Essais sur la disparition de la matière sèche in vitro}

Ces essais ont été réalisés à la Station d'Amélioration des Plantes fourragères de Lusignan (I. N. R. A.), sur des graines issues de croisements génétiques encore assez mal définis. IJa digestibilité de la matière sèche in vitro a été déterminée par la méthode de Tilley et Terry (r963) avec les modifications apportées par Alexander et McGowan (I966).

La méthode est caractérisée par deux étapes :

- une digestion cellulolytique, réalisée en mettant en présence, pendant $4^{8}$ heures, l'échantillon d'une part et un jus de Rumen d'autre part ;

- une digestion protéolytique faite en traitant le résidu de la première digestion par une solution de pepsine en milieu acide. L'incubation dure 48 heures.

A l'issue de la seconde étape, le résidu de digestion est recueilli. Le poids de ce résidu est corrigé par l'introduction de "blancs" dans la série (blanc $=$ tampon + jus de Rumen). Le coefficient de digestibilité de la matière sèche est défini par l'expression :

$$
\mathrm{CD}_{\mathrm{MS}}=\frac{\text { Quantité de départ }- \text { résidu réel }}{\text { Quantité de départ }}
$$

résidu réel $=$ résidu apparent — blanc. 
Outre les blancs, on introduit dans chaque série un certain nombre de standards. Le standard est un échantillon de fourrage de digestibilité in vivo connue qui permet de corriger les variations d'activité du jus de Rumen dans le temps et dans la série de mesure.

\section{RÉSULTATS ET DISCUSSION}

\section{A. - Structure chimique des tanins présents dans les graines de sorgho}

Les téguments de la plupart des graines de sorgho renferment des tanins condensés qu'on peut encore appeler proanthocyanidines. Ces polymères proviennent de la condensation de molécules de flavanes; deux motifs élémentaires semblent impliqués dans ces structures complexes :

- l'épicatéchine : 5, 7, 3', 4' - tétrahydroxyflavane 3-ol (I);

- la leucocyanidine : 5, 7, 3', 4' - tétrahydroxyflavane 3-4 diols (II).

Chaque fraction $A, B$ et $C$ des proanthocyanidines du sorgho libère de la cyanidine (III) par chauffage prolongé avec un acide fort, et produit lors d'une hydrolyse ménagée avec un acide faible, un flavanol-3 qui est l'épicatéchine (I), et trois dimères formés chacun par une molécule d'épicatéchine et par une molécule de leucocyanidine (IV). Ces dimères encore appelés biflavanes libèrent de la cyanidine lors d'une hydrolyse forte et de l'épicatéchine au cours d'une hydrolyse ménagée. Ils possè-

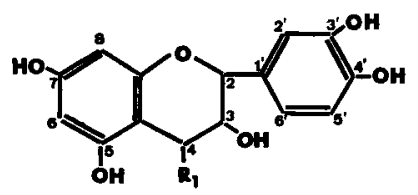

I. $\mathbf{R}_{\mathbf{1}}=\boldsymbol{H}$, épicatechine

$(5,7,3,4$ - tètrohydroxyflavane -3 ol $]$

Prósence d'un carbone asymétrique

en 2 of 3

II. $R_{1}=\mathbf{O H}$, leucocyanidine

(s,7,3,4-1étrahydroxyflavane_3,4 diols)

Presence d'un carbone asymétrique

en 2,3 et 4<smiles>OC1=c2cc(O)c3cc2=C(c2ccc(O)c(O)c2)OC(=C1)O3</smiles>

III. cyanidine

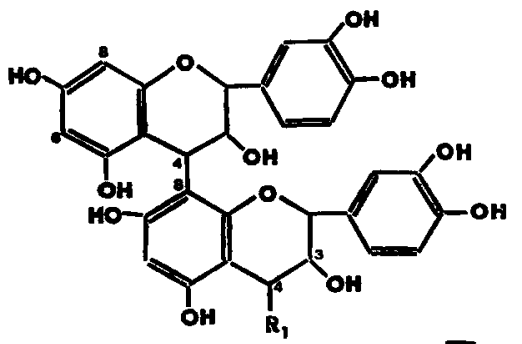

IY. $R_{1}=H$, biflevane

(loucocyanidine, epicatéchine) 


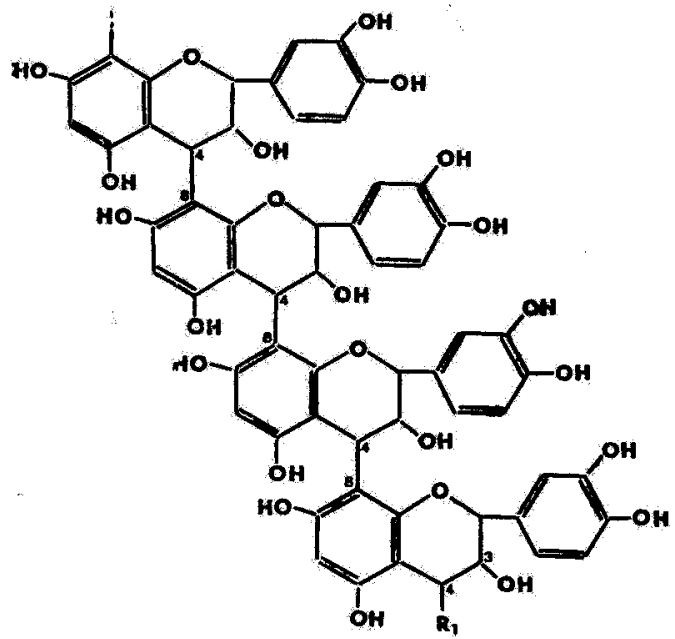

I. $R_{y}=H$, procyenidine du sorgho grain

(n. molécules de leucocyanidine, epicaléchine)<smiles></smiles>

ZI. $R_{2}=H$, apigoninidino

D. $R_{2}=\mathrm{OH}$, Iuteolinidino<smiles>O=C(O)c1cc(C2C[C@H](O)c3c(O)cc(O)cc3O2)ccc1O</smiles>

7.1. $\mathbf{R}_{2}=H$, teucoopleoninidime

[s,7,4-trithydranyflavone - ad)

R. $R_{2}=O H$, leucoluteolinidine

(3,7,3\%'-totrahydroxyl buane-4ol) 
dent des $\mathrm{R} f$ différents dans les solvants utilisés. Ces composés paraissent être des stéréoisomères (présence de carbones asymétriques dans la molécule de leucocyanidine et dans celle de l'épicatéchine).

Les biflavanes du sorgho sont comparables à celles isolées des graines de Persea gratissima (GeIssman et DitTMAR, I965) des feuilles de Fragaria Vesca (CREAsy et Swain, I965) et des graines de Theobroma cacao (QUESNEL, I968). Elles possèdent les mêmes propriétés physicochimiques.

Les proanthocyanidines présentes dans les graines de nombreuses variétés de sorgho seraient formées par des molécules de leucocyanidine, chaque chaîne se terminant par une unité épicatéchine ; ce sont donc plus précisément des procyanidines ; les liaisons entre molécules s'effectueraient comme chez la biflavane entre le carbone 4 d'une molécule et le carbone 6 ou 8 de la suivante (V). Les molécules de flavanes possédant plusieurs carbones asymétriques, le nombre d'isomères possibles est considérable.

Les fractions B mais surtout C des procyanidines du sorgho donnent un précipité important après addition d'une solution de gélatine. Ces polymères semblent posséder des propriétés tannantes.

Les téguments des graines de sorgho dépourvus de procyanidines contiennent en grande quantité des leucoanthocyanidines peu polymérisées libérant par chauffage en présence d'un acide fort des anthocyanidines comme la lutéolinidine (VII) $(\lambda \max 496 \mathrm{~nm})$ et à un moindre degré l'apigéninidine (VI) $(\lambda \max 476 \mathrm{~nm}$ ). Le chauffage de tels extraits en milieu acide conduit à la formation d'une coloration cerise devenant rapidement orange.

Les flavanes sont dans ce cas des flavanols-4 : la leucolutéolinidine ou lutéoforol (IX) $\left(5,7,3^{\prime}, 4^{\prime}\right.$ - tétrahydroxyflavane-4 ol), et la leucoapigéninidine (VIII) (5, $7,4^{\prime}$ - trihydroxyflavane-4 ol).

La présence de telles substances a été notée par BAtre-Smith et Swain (I967) dans les feuilles de graminées de la tribu des Andropogoneae.

BATE-Smith (I969) a également identifié du lutéoforol dans les téguments de certaines graines de sorgho.

\section{B. - Effets des tanins condensés sur l'utilisation digestive du sorgho-grain chez le Rat en croissance (tabl. 3)}

La digestibilité de l'énergie des régimes varie de 79 à $90 \mathrm{p}$. Ioo ; cette variation sst très importante pour une céréale donnée. La digestibilité de l'azote du régime varie considérablement : de 50 à $88 \mathrm{p}$. Ioo. Le classement adopté đans le tableau 3 et la figure $\mathrm{I}$ font apparaître que les régimes correspondant à des échantillons de sorgho qui ont une teneur très faible en procyanidines, ont des CUDa élevés pour l'énergie et surtout pour l'azote. Inversement, les échantillons qui ont des teneurs élevées en procyanidines correspondent à des CUDa plus faibles pour l'énergie et surtout pour l'azote. La diminution de la digestibilité en fonction de la teneur en procyanidines est linéaire (fig. I).

Ces premiers résultats montrent que ce sont les tanins peu polymérisés (sorghos AKS 6I4 et INRA 450, I97I) qui ont une action dépressive sur 1'utilisation digestive des matières azotées.

Les tanins doivent agir aussi bien sur la digestibilité des matières azotées du 
TABIEAU 3

Effets des proanthocyanidines sur "utilisation digestive du Sorgho grain chez le Rat in croissance

\begin{tabular}{|c|c|c|c|c|c|c|c|}
\hline Échantillon & INRA 450 & NK 222 & INRA 450 & INRA 450 & NK 120 & INRA 450 & AKS 614 \\
\hline $\begin{array}{l}\text { Année } \\
\text { Origine }\end{array}$ & $\begin{array}{r}1967 \\
\text { C.A.L. }\end{array}$ & $\begin{array}{c}1970 \\
\text { E.N.S.A. }\end{array}$ & $\begin{array}{c}1971 \\
\text { E.N.S.A. }\end{array}$ & $\begin{array}{c}1970 \\
\text { E.N.S.A. }\end{array}$ & $\begin{array}{r}1970 \\
\text { C.A.L. }\end{array}$ & $\begin{array}{r}1971 \\
\text { C.A.L. }\end{array}$ & $\begin{array}{c}1970 \\
\text { E.N.S.A. }\end{array}$ \\
\hline LA (teneur en procyanidines) & 0,01 & 0,05 & 0,31 & 0,85 & 0,375 & 0,45 & 0,60 \\
\hline $\mathrm{V}, \ldots \ldots \ldots \ldots \ldots \ldots \ldots$ & $\rightarrow$ & - & 0,14 & 0,145 & 0,17 & 0,25 & 0,48 \\
\hline $\begin{array}{l}\text { V/LA } \ldots \ldots \ldots \ldots \ldots \ldots \ldots \\
\text { Énergie brute ingérée }\end{array}$ & - & 一 & 0,45 & 0,41 & 0,45 & 0,55 & 0,80 \\
\hline $\begin{array}{c}(\mathrm{kcal} / \mathrm{rat} / \mathrm{j}) \ldots \ldots \ldots \ldots \ldots \\
\text { Énergie métabolisable ingérée }\end{array}$ & 66,7 & 68,8 & 65,7 & 64,5 & 65,6 & 71,0 & 75,3 \\
\hline (kcal/rat $/ \mathrm{j}) \ldots \ldots \ldots \ldots \ldots$ & 58,0 & 60,8 & 54,0 & 52,1 & 52,4 & 56,5 & 58,1 \\
\hline$N$ ingéré $(\mathrm{mg} / \mathrm{rat} / \mathrm{j}) \ldots \ldots \ldots$ & 383 & 308 & 340 & 317 & 321 & 389 & 448 \\
\hline $\mathbf{N}$ absorbé $(\mathrm{mg} / \mathrm{rat} / \mathrm{j}) \ldots \ldots$ & 333 & 250 & 210 & 204 & 174 & 226 & 222 \\
\hline $\mathrm{N}$ retenu $(\mathrm{mg} / \mathrm{rat} / \mathrm{j}) \ldots \ldots$. & 174 & 167 & 147 & 116 & 108 & 139 & 130 \\
\hline CUDa $\mathrm{E} \ldots \ldots \ldots \ldots \ldots$ & 89,8 & 90,1 & 83,5 & 83,1 & 81,1 & 81,5 & 79,0 \\
\hline Régime EM p. $100 \mathrm{EI} \ldots$. & 87,0 & 88,3 & 82,2 & 81,4 & 79,7 & 79,6 & 77,2 \\
\hline CUDa $\mathrm{N} \ldots \ldots \ldots \ldots \ldots \ldots$ & 87,7 & 81,3 & 61,9 & 64,2 & 54,1 & 58,1 & 49,5 \\
\hline CUP $\mathrm{N}$ fixé $\times 100 / \mathrm{N}$ ingéré .. & 45,4 & 54,3 & 43,3 & 36,4 & 34,9 & 35,7 & 29,0 \\
\hline CR $N$ fixé $\times 100 / \mathrm{N}$ absorbé. & 52,3 & 66,8 & 69,9 & 57,1 & 64,5 & 61,4 & 58,6 \\
\hline
\end{tabular}

Les grandeurs LA et $\mathrm{V}$ sont exprimées en densités optiques et mesurées pour une concentration de $8 \mathrm{mg}$ de poids frais de graines.

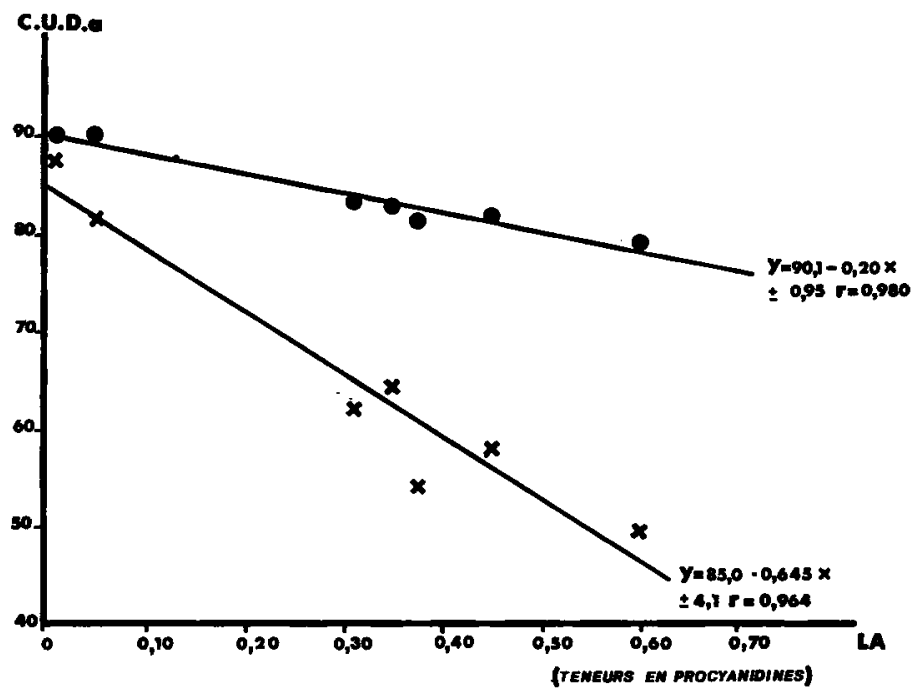

FIG. I. - Influence des teneurs en procyanidines du sorgho sur les CUDa de l'énergie (•) et de l'azote (x) des régimes expérimentaux chez le Rat en croissance

(Les teneurs en procyanidines sont exprimées en densités optiques par la grandeur IA et correspondent à $8 \mathrm{mg}$ de poids frais de graines) 
sorgho que sur celle des autres sources d'azote du régime (farine de poisson) et de 1'azote endogène, en particulier les protéines des sécrétions digestives.

De plus, les tanins semblent réduire l'utilisation métabolique des acides aminés absorbés. En effet, la rétention azotée est faible (I ro à $\mathrm{I} 40 \mathrm{mg} \mathrm{N} / \mathrm{rat} / \mathrm{j}$ ) malgré les quantités d'azote absorbées assez élevées ( 174 à $226 \mathrm{mg} / \mathrm{j}$ ). Compte tenu des quantités d'énergie métabolisables ingérées dans les différents lots et de 1a supplémentation en acides aminés indispensables qui a été faite (farine de poissons, acides aminés industriels) on pouvait attendre une rétention azotée et un coefficient de rétention azoté ( $\mathrm{N}$ fixé $\times$ Ioo/ $\mathrm{N}$ absorbé) beauc.sup plus élevés.

On peut supposer qu'une partie importante des acides aminés indispensables du régime et des protéines digestives a été bloquée par les tanins donc non digérée et rendue indisponible pour l'organisme du Rat en croissance.

\section{C. - Effet des tanins condensés sur la "digestibilité in vitro " de la matière sèche du sorgho}

Des essais préliminaires ont été réalisés sur des hybrides actuellement en cours d'étude (LENOBLE, Station d'Amélioration des Plantes fuurragères, I. N. R. A. Lusignan). Comme le montre la figure 2, il existe une étroite corrélation entre les quantités de procyanidines présentes dans les graines de sorgho et la « digestibilité in vitro » de la matière sèche.

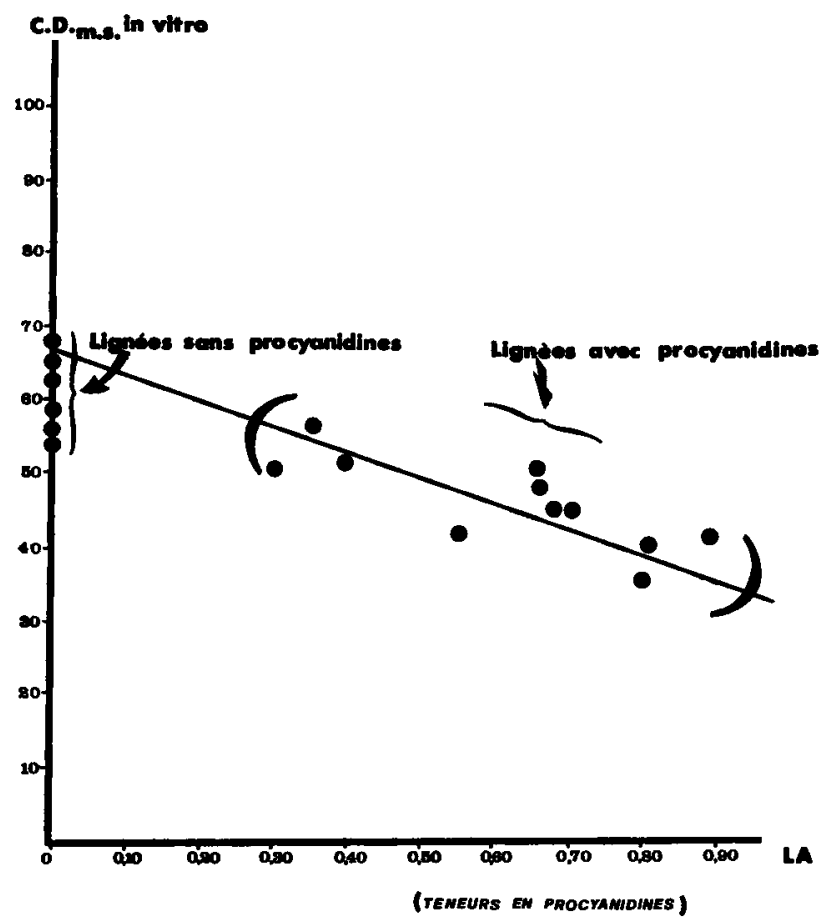

FIG. 2. - Influence des procyanidines sur le coefficient de digestibilite de la matière sèche (CDms), in vitro, dans le sorgho grain

(Les quantités en procyanidines sont exprimées par la grandeur LA et correspondent à $8 \mathrm{mg}$ de poids frais de graines) 
Dans tous les.cas, la présence de procyanidines (proportionnelle à la valeur IAA) intervient proportionnellement sur la disparition de la matière sèche.

Par contre, les lignées nouvelles dépourvues de procyanidines, mais riches en leucoanthocyanidines libérant de la lutéolinidine et de l'apigéninidine interfèrent le moins avec la disparition de la matière sèche in vitro. La dispersion des points sur la ligne des ordonnées peut avoir deux significations :

- d'autres facteurs interfèrent avec les tanins dans ces expériences in vitro ;

- le test de "digestibilité in vitro de la matière sèche " est de maigre valeur pour l'étude du rôle des tanins dans la nutrition des polygastriques.

\section{CONCLUSION}

Ces résultats montrent que la présence de procyanidines est corrélative d'une importante diminution de l'utilisation digestive des matières azotées chez le Rat en croissance et ont un effet dépressif sur la disparition de la matière sèche in vitro. Les leucoanthocyanidines peu polymérisées et dont les molécules constitutives sont le lutéoforol et la leucoapigéninidine n'apparaissent pas comme responsables de tels effets.

Le prochain travail consistera à étudier les propriétés physicochimiques des différentes fractions de procyanidines isolées de graines de certaines variétés de sorgho. Les fractions seront incorporées au régime alimentaire de rats en croissance. I1 sera alors possible d'établir une relation entre structure des tanins et digestibilité de l'azote.

Reçu pour publication en mars 1976.

\section{SUMMARY}

\section{IMPORTANCE OF SORGHUM SEED TANNINS IN PROTEIN CONVERSION IN GROWING RATS}

Condensed tannins or proanthocyanidins formed from leucocyanidin are found in the seedcoats of most varietes of sorghum. They inhibit protein conversion in growing rats and have a depressive effect in vitro on dry matter disappearance after " enzymatic digestion $\nu$.

\section{RÉFÉRENCES BIBLIOGRAPHIQUES}

Alexander R. M., Mac Gowan M., I966. The routine determination of in vitro digestibility of organic matter in forages an investigation of the problems associated with continuous large scale operation. J. Brit. Grasse. Soc., 21, 140-147.

Bate-Smith E. C., Swain T., 1967. New leuco-anthocyanins in grasses. Nature, 213, ro33-ro34.

Bate-Smith E. C., I969. Luteoforol $\left(3^{\prime}, 4^{\prime}, 4^{\prime}, 5,7\right.$-pentahydroxyflavan) in Sorghum vulgave L. Phytochemistry, 8, 1803-1810. 
Bate-Smith E. C., I973. Haemanalysis of tannins : the concept of relative astringency. Phytochemistry, 12, 907-912.

Bate-Smith E. C., I973. Tannins of herbaceous leguminosae. Phytochemistry, 12, I809-I812.

Chang S. I., Fuller H. L., 1964. Effect of tannin content of grain sorghums on their feeding value for growing chicks. Poult. Sci., 43, 30-36.

Creasy L. L., Swain T., I965. Structure of condensed tannins. Nature, 208, I5I-I53.

Geissman T. A., Ditrmar H. F, K., I965. Proanthocyanidin from avocado seed. Phytochemistry, 4, 359-368.

McGinty D. D., I968. Factors affecting the digestibility of varieties of sorghum grain by cattle. $\mathrm{Ph}$. D. Thesis. Texas A et M University College Station, Texas.

Goldstein J., Swain T., 1963. Changes in tannins in ripening fruits. Phytochemistry, 2, 371-383.

Harris H. B., 1969. Bird resistance in sorghum. Ann. Corn and Sorghum Res. conf., Proc. 24th (Chicago, Ill.), II3-I22.

QUESNEL V. C., 1968. Fractionation and properties of the polymeric leucocyanidin of the seeds of Theobroma cacao. Phytochemistry, 7, 1583-1592.

Swain T., Hrulis W. E., r959. The phenolic constituents of Prunus domestica. The quantitative analysis of phenolic constituents. J. Sci. Foof. Agric., 10, 63-68.

Swarn T., Goldstein J. L., I964. In Methods in Polyphenol chemistry. J. B. Pridham editeur. Pergamon Press, Oxford., 9-34.

Tilley J. M. A., TeRRY R. A., I963. A Two stage technique for the in vitro digestion of forage crops. J. Brit. Grasse. Soc., 18, I04-III.

Troyer J. R., 1964. Leucoanthocyanin formation in buckwheat seedling hypocotyls. Phytochemistry, 8, 535-539.

VAN BRAGT J., 1966. Dunne-laag chromatografie, toegepast bijhet onderzoek van bloemkleurstoffen. Meded. Dis. Tuinb. (Februari), 2, 97-99.

Vermorel M., I97o. Utilisation de l'énergie et de l'azote du sorgho hybride " I. N. R. A. 450 " composant un régime équilibré en acides aminés indispensables par le Rat en croissance. Ann. Biol. anim Bioch. Biophys., 10, 327-330.

Vermorel M., 1973. Utilisation énergétique et azotée des principaux tourteaux et des graines de légumineuses par le Rat en croissance; comparaison au blé tendre et au blé dur. Ann. Zootech., 22, 253265. 